\section{ASPECTOS BIOLÓGICOS DIFERENCIALES EN EL CÁNCER SEGÚN EL GÉNERO}

\author{
Fernando López-Ríos \\ Hospital Universitario HM Sanchinarro \\ flopezrios@hmhospitales.com
}

Cómo citar este artículo/Citation: López-Ríos, F. (2015). "Aspectos biológicos diferenciales en el cáncer según el género". Arbor, 191 (773): a233. doi: http://dx.doi. org/10.3989/arbor.2015.773n3003

Recibido: 9 diciembre 2014. Aceptado: 24 abril 2015.

RESUMEN: Diferentes trabajos muestran que las mujeres son potencialmente más vulnerables a desarrollar un cáncer de pulmón (CP). En particular, las mujeres son más propensas a desarrollar adenocarcinomas, suelen ser más jóvenes, son diagnosticadas en estadios más iniciales y poseen un mayor riesgo las no fumadoras. Por otra parte, no en todos los CP existe una causa concreta detectada, lo que hace pensar en la existencia de efectos aditivos y sinérgicos entre distintas causas, así como en la existencia de factores de predisposición y de riesgo para padecer un CP. En este sentido, la hipótesis de que los estrógenos pueden desempeñar un papel en la carcinogénesis del $C P$ va cobrando importancia, aunque el mecanismo de esta implicación no está claro. Aunque todo lo expuesto en esta revisión sugiere que las mujeres son más susceptibles al tabaco y potencialmente más vulnerables a desarrollar un $\mathrm{CP}$, la influencia de estas características diferenciales en cuanto a la supervivencia es controvertida.

\section{GENDER-RELATED BIOLOGICAL DIFFERENCES IN CANCER}

Copyright: (c) 2015 CSIC. Este es un artículo de acceso abierto distribuido bajo los términos de la licencia Creative Commons Attribution-Non Commercial (by-nc) Spain 3.0.

ABSTRACT: Several studies show that women are potentially more susceptible to developing lung cancer (LC). Specifically, women are more prone to adenocarcinomas, they usually develop the disease younger, are diagnosed at earlier stages, and female non-smokers are at higher risk. On the other hand, no particular cause has been detected for all LC, which suggests the presence of additive synergistic effects among several causes, in conjunction with predisposing and risk factors for LC. In line with this, the hypothesis that oestrogens can play a role in LC carcinogenesis is gaining acceptance, although the mechanism by which oestrogens are involved in this process is not clear. Although, on the whole, this review suggests women are more likely to smoke and so potentially more prone to develop LC, the influence of these differential characteristics regarding survival is controversial.

KEYWORDS: estrogens; lung cancer; tobacco. 
De entre todos los cánceres, es el cáncer de pulmón (CP) el que posee más aspectos diferenciales según el género. Actualmente, parece bastante establecido que existen importantes diferencias biológicas entre hombres y mujeres en relación al riesgo de padecer esta enfermedad. Diferentes trabajos muestran que las mujeres son potencialmente más vulnerables a desarrollar un CP. En particular, las mujeres son más propensas a desarrollar adenocarcinomas (ACs), suelen ser más jóvenes, son diagnosticadas en estadios más iniciales y poseen un mayor riesgo las no fumadoras (Kiyohara y Ohno, 2010; Pesatori et al., 2013).

En relación a la sensibilidad al tabaco, las diferencias entre hombres y mujeres han sido bien documentadas (Kiyohara y Ohno, 2010). Algunos trabajos han descrito diferencias estadísticamente significativas para los principales tipos de CP, siendo mayor el riesgo de padecer un $\mathrm{CP}$ en las mujeres fumadoras que en los hombres independientemente de los niveles de exposición al humo del tabaco. Los resultados de estos estudios no se pueden explicar por las diferencias en la exposición inicial, en el tiempo de tabaquismo o en el tamaño corporal, si no que los autores describen que son probablemente debidas a una mayor susceptibilidad a los carcinógenos del tabaco en las mujeres (Zang y Wynder, 1996). Por otra parte, estudios de mutaciones de TP53 en relación con el tabaquismo y el sexo han demostrado que los CP que aparecen en mujeres fumadoras tienen significativamente más mutaciones relacionadas con el tabaco ( $\mathrm{C}>\mathrm{T}$ transversiones) que los hombres fumadores (Marrogi et al., 2005). Asimismo, un reciente trabajo ha observado un mayor porcentaje de mujeres con tumores con mutación KRAS G12C (debida también a transversiones $\mathrm{G}$ $>\mathrm{T}$ ), siendo más jóvenes al diagnóstico y con menor número de paquetes-años de tabaquismo en comparación con los hombres con la misma alteración, proporcionando otros datos que apoyan la hipótesis de que las mujeres son más susceptibles a los carcinógenos del tabaco (Dogan et al., 2012).

Esta mayor susceptibilidad puede reflejar diferencias constitutivas de genes que codifican enzimas que metabolizan los carcinógenos del tabaco. Por ejemplo, la enzima CYP1A1 (o P5401A1, fase I del citocromo P450), que cataliza el primer paso en el metabolismo de los hidrocarburos aromáticos policíclicos (HAPs) (componentes del tabaco), muestra una mayor expresión en el parénquima pulmonar normal de las mujeres fumadoras respecto al de los hombres fumadores. Además, el polimorfismo más frecuente observado en el citocromo P450 es el genotipo GSTM1-null (fase II del citocromo P540S, responsable de la detoxificación de las formas activas de HAPs), que está presente en el $40-50 \%$ de la población general, pudiendo aumentar el impacto de este genotipo GSTM1-null en mujeres fumadoras (Kiyohara y Ohno, 2010). También se han descrito en mujeres niveles superiores de aductos de HAPs en comparación con los hombres, incluso con menores niveles de carcinógenos del tabaco (Kiyohara y Ohno, 2010). Estos hallazgos sugieren que la susceptibilidad al daño del ADN provocada por componentes del tabaco tipo HAPs es mayor en mujeres que en hombres, a pesar de fumar significativamente menos. Igualmente, se ha descrito que las mujeres tienen una menor capacidad de reparación del ADN y, por tanto, un mayor riesgo de padecer un CP (Kiyohara y Ohno, 2010) (Figura 1).

Aunque el tabaco es el factor etiológico fundamental en el $\mathrm{CP}$, existen otros carcinógenos relacionados fundamentalmente con exposiciones ocupacionales (asbestos, radón, arsénico, entre otros) que pueden considerarse responsables del $1 \%$ de los CP de mujeres (Cabanes et al., 2010). Por otra parte, no en todos los CP existe una causa concreta detectada, ni la presencia de un agente etiológico conlleva siempre la aparición de un $\mathrm{CP}$, lo que hace pensar en la existencia de efectos aditivos y sinérgicos entre distintas causas, así como en la existencia de factores de predisposición y de riesgo para padecer un CP. En este sentido, la hipótesis de que los estrógenos pueden desempeñar un papel en la carcinogénesis del CP va cobrando importancia. No obstante, el mecanismo por el cual los estrógenos parecen estar implicados en la carcinogénesis pulmonar no está claro. Algunos investigadores han descrito que pueden actuar como ligandos de receptores (ER, receptores de estrógenos) y activar la proliferación celular y/o estimular la angiogénesis, la cual está asociada al potencial desarrollo de metástasis (Losordo e Isner, 2001). Recientemente, otros trabajos han demostrado que terapias anti-estrógenos pueden reducir el riesgo de muerte por CP (Stabile et al., 2011). Por el contrario, otros estudios sugieren un posible papel protector de los estrógenos endógenos, mostrando un menor riesgo de padecer un CP en mujeres con menopausia tardía y largo periodo reproductivo (Pesatori et al., 2013).

Los estrógenos actúan en la célula a través de la unión a sus receptores (ER), que pertenecen a la familia de los factores de transcripción conocidos como receptores nucleares. Una vez unido al ligando, el receptor experimenta cambios conformacionales que le permiten dimerizar y unirse a los elementos de 
Figura 1. Propuesta de la vía de la carcinogénesis pulmonar en mujeres

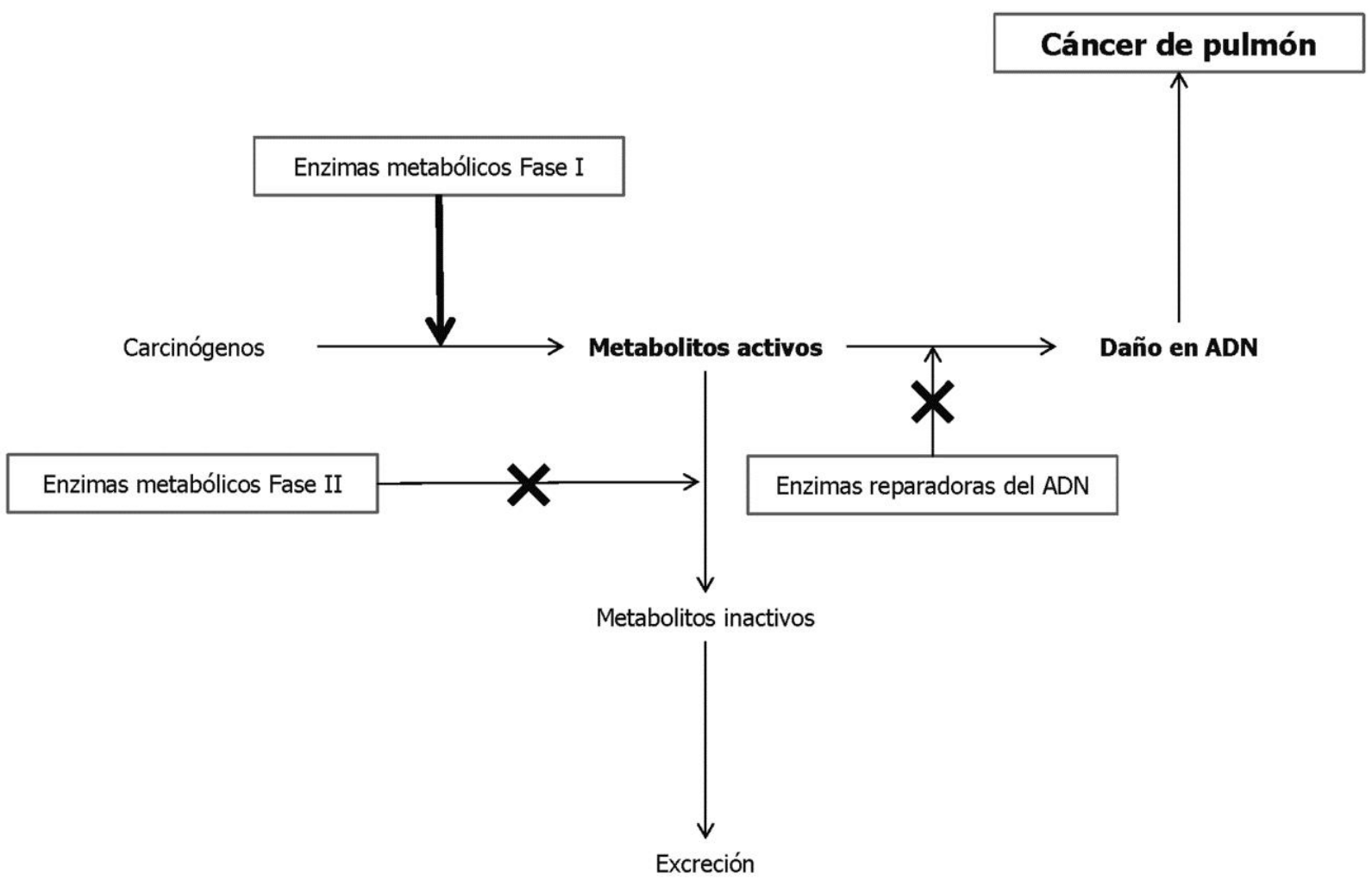

Modificado de Kiyohara et al. 2010.

Una mayor expresión en mujeres de las enzimas Fase I del citocromo P450, responsables del metabolismo de los carcinógenos del tabaco, junto a una menor actividad de las enzimas Fase II, responsables de la detoxificación de los metabolitos activos, sugieren una mayor susceptibilidad al daño del ADN. Asimismo, la menor capacidad de reparación del ADN descrita, contribuyen también al riesgo de padecer un CP en las mujeres.

respuesta a estrógenos presentes en sus genes diana regulando la expresión génica. En 1996, se descubrió la existencia de un segundo subtipo de receptor, llamado $E R \beta$, para distinguirlo del receptor estrogénico conocido hasta ese momento $(E R \alpha)$. Estos receptores están codificados por genes distintos y presentan importantes diferencias en cuanto a su expresión y distribución tisular. ER $\alpha$ se expresa principalmente en útero, hígado, riñón y corazón, mientras que ERß se expresa en ovario, próstata, pulmón, tracto gastrointestinal, vejiga, sistema hematopoyético y sistema nervioso central (Matthews y Gustafsson, 2003). Estudios con animales knock-out (KO) para ER $\beta$ han puesto de manifiesto un importante papel de este receptor en el pulmón, ya que en ratones hembras de este tipo se aprecian a los 3 meses anormalidades en los pulmones. A los 5 meses de edad, los animales ER $\beta$ KO de ambos sexos muestran signos evidentes de disfunción pulmonar (Patrone et al., 2003). Cabe destacar que se ha observado una localización nu- clear de ER $\beta$ en el $45-69 \%$ de los casos de CPNM, demostrándose en algunos estudios que es un indicador de pronóstico favorable (Hershberger et al., 2009). El análisis y la interpretación de la positividad de ER $\alpha$ en CPNM son considerablemente más complejos. La detección nuclear de ER $\alpha$ suele ser inexistente o escasa en CP primarios (Hershberger et al., 2009), observándose más frecuentemente expresión citoplasmática en una proporción que varía ampliamente del 3\% al 73\% (Wu et al., 2005).

Las diferencias de predominancia en ER $\alpha$ o ER $\beta$ en los distintos tejidos, pueden contribuir a explicar la controversia existente sobre los efectos protectores de los estrógenos en ciertos tejidos, en los que suele predominar ERß (como el pulmón), a la vez que suponen un riesgo para el desarrollo del cáncer en otros tejidos, tejidos relacionados con el sistema reproductor, en los cuales suele predominar ER $\alpha$ (Morani, Warner y Gustafsson, 2008). 
Aunque todo lo expuesto sugiere que las mujeres son más susceptibles al tabaco y potencialmente más vulnerables a desarrollar un $\mathrm{CP}$, la influencia de estas características diferenciales en cuanto a la supervivencia es controvertida (Kiyohara y Ohno, 2010).

\section{BIBLIOGRAFÍA}

Cabanes, A., Vidal, E., Aragones, N., Perez-Gomez, B., Pollan, M., Lope, V., et al. (2010). Cancer mortality trends in Spain: 1980-2007. Annals of Oncology, 21, Suppl 3, pp. iii14-20. DOI: mdq089 [pii]10.1093/annonc/mdq089.

Dogan, S., Shen, R., Ang, D. C., Johnson, M. L., D’Angelo, S. P., Paik, P. K., et al. (2012). Molecular epidemiology of EGFR and KRAS mutations in 3,026 lung adenocarcinomas: higher susceptibility of women to smoking-related KRAS-mutant cancers. Clinical Cancer Research, 18, 22, pp. 6169-6177. DOI: 1078-0432.CCR-11-3265 [pii]10.1158/1078-0432.CCR-11-3265.

Hershberger, P. A., Stabile, L. P., Kanterewicz, B., Rothstein, M. E., Gubish, C. T., Land, S., et al. (2009). Estrogen receptor beta (ERbeta) subtype-specific ligands increase transcription, p44/p42 mitogen activated protein kinase (MAPK) activation and growth in human nonsmall cell lung cancer cells. Journal of Steroid Biochemistry and Molecular Biology, 116, 1-2, pp. 102-109. DOI: S0960-0760(09)00158-7 [pii]10.1016/j. jsbmb.2009.05.004.

Kiyohara, C. y Ohno, Y. (2010). Sex differences in lung cancer susceptibility: a review. Gender Medicine, 7, 5, pp. 381-401. DOI: S1550-8579(10)00102-6 [pii]10.1016/j.genm.2010.10.002.
Losordo, D. W. e Isner, J. M. (2001). Estrogen and angiogenesis: A review. Arteriosclerosis, Thrombosis and Vascular Biology, 21, 1, pp. 6-12.

Marrogi, A. J., Mechanic, L. E., Welsh, J. A., Bowman, E. D., Khan, M. A., Enewold, L., et al. (2005). TP53 mutation spectrum in lung cancer is not different in women and men. Cancer Epidemiology, Biomarkers and Prevention, 14, 4, pp. 1031-1033. DOI: 14/4/1031 [pii]10.1158/1055-9965.EPI-04-0640.

Matthews, J. y Gustafsson, J. A. (2003). Estrogen signaling: a subtle balance between ER alpha and ER beta. Molecular Interventions, 3, 5, pp. 281-292. DOI: 10.1124/mi.3.5.2813/5/281 [pii].

Morani, A., Warner, M. y Gustafsson, J. A. (2008). Biological functions and clinical implications of oestrogen receptors alfa and beta in epithelial tissues. Journal of Internal Medicine, 264, 2, pp. 128142. DOI: JIM1976 [pii]10.1111/j.13652796.2008.01976.x

Patrone, C., Cassel, T. N., Pettersson, K., Piao, Y. S., Cheng, G., Ciana, P., et al. (2003). Regulation of postnatal lung development and homeostasis by estrogen receptor beta. Molecular and Cellular Biology, 23, 23, pp. 8542-8552.
Pesatori, A. C., Carugno, M., Consonni, D., Caporaso, N. E., Wacholder, S., Tucker, M., et al. (2013). Reproductive and hormonal factors and the risk of lung cancer: the EAGLE study. International Journal of Cancer, 132, 11, pp. 2630-2639. DOI: 10.1002/ijc.27926.

Stabile, L. P., Dacic, S., Land, S. R., Lenzner, D. E., Dhir, R., Acquafondata, M., et al. (2011). Combined analysis of estrogen receptor beta-1 and progesterone receptor expression identifies lung cancer patients with poor outcome. Clinical Cancer Research, 17, 1, pp. 154-164. DOI: 1078-0432.CCR-10-0992 [pii]10.1158/1078-0432.CCR-10-0992.

Wu, C. T., Chang, Y. L., Shih, J. Y. y Lee, Y. C. (2005). The significance of estrogen receptor beta in 301 surgically treated non-small cell lung cancers. Journal of Thoracic and Cardiovascular Surgery, 130, 4, pp. 979-986. DOI: S0022-5223(05)00979-7 [pii]10.1016/j. jtcvs.2005.06.012.

Zang, E. A. y Wynder, E. L. (1996). Differences in lung cancer risk between men and women: examination of the evidence. Journal of the National Cancer Institute, 88, 3-4, pp. 183-192. 